\title{
La intervención social mediante proyectos comunitarios en modalidad virtual en Mérida, México. Una perspectiva desde el Trabajo Social
}

\author{
Claudia Isabel Tzec-Puch
}

Magister en Ciencias del Movimiento Humano. Trabajadora social

Universidad Autónoma de Yucatán. Mérida, México

https:/ / orcid.org/0000-0001-6996-6792 • claudia.tzec@correo.uady.mx

\section{Amairani Aracelly Ceh-Alvarado}

Magister en Ciencias del Movimiento Humano. Trabajadora social

Universidad Autónoma de Yucatán. Mérida, México

https://orcid.org/0000-0002-4340-5724 • amairani.ceh@correo.uady.mx

\section{Yanet Guadalupe González-Canul}

Magister en Ciencias del Movimiento Humano. Trabajadora social

Universidad Autónoma de Yucatán. Mérida, México

https:/ / orcid.org/0000-0002-6792-4000 • yanet.gonzalez@correo.uady.mx

\section{Resumen}

Este trabajo tiene como propósito recuperar y sistematizar los aprendizajes profesionales de la Licenciatura en Trabajo Social de la Universidad Autónoma de Yucatán, del campo práctico en comunidades que corresponde a la etapa de intervención del proceso metodológico de Trabajo Social, que por primera vez se llevó a cabo en modalidad virtual, debido al distanciamiento social causado por la COVID-19.

El estudio se basó en la propuesta metodológica de Castañeda, la cual comprende cinco fases: contextos de la experiencia, referentes teóricos conceptuales, decisiones metodológicas, desarrollo del proceso de análisis, reflexiones, aprendizajes y conclusiones del proceso. Asimismo, se empleó la revisión documental a través de los siguientes instrumentos: proyectos de intervención, cartas descriptivas, crónicas grupales, reportes e informes de supervisión y programa operativo. La validación de la información se llevó a cabo por el juicio de expertos.

Como resultado se identificaron los roles del trabajador social: educador social, animador sociocultural y gestor social; además de la creación de espacios virtuales de difusión, participación e interacción con la población y finalmente el rigor de la metodología empleada. A modo de conclusión, el proceso metodológico de la práctica comunitaria, integrada en cinco etapas sustanciales, enmarcadas en la virtualidad, contribuyó al desarrollo de competencias profesionales, dando respuesta al contexto de la nueva realidad.

Palabras clave: Sistematización; Intervención; Proyecto virtual; Comunidad.

Recibido: 07/07/2021 | Aprobado: 28/08/2021 | Publicado: 01/01/2022

(i) (-) Esta obra está bajo una Licencia Creative Commons Atribución-NoComercial-CompartirIgual 4.0 Internacional.

Financiación o proveniencia del artículo: Artículo derivado de la Sistematización de experiencias del campo práctico en comunidades, de la Licenciatura en Trabajo Social, Universidad Autónoma de Yucatán, 2021.

\section{¿Cómo citar este artículo? / How to quote this article?}

Tzec-Puch, C. I., Ceh-Alvarado, A. A., y González-Canul, Y. G. (2022). La intervención social mediante proyectos comunitarios en modalidad virtual en Mérida, México. Una perspectiva desde el Trabajo Social. Prospectiva. Revista de Trabajo Social e intervención social, (33), 117-141. doi: 10.25100/prts.v0i33.11440. 


\title{
Social Intervention Through Community Projects in Virtual Mode in Merida, Mexico. A Perspective from Social Work
}

\begin{abstract}
The purpose of this work is to gather and systematize the professional learning of the Bachelor in Social Work at the Universidad Autónoma de Yucatán. The learning experiences are from the practical work in communities, which for the first time was carried out in virtual mode due to the social distancing caused by COVID-19.

The study was based on Castañeda's methodological proposal, which consists of five phases: experience contexts, conceptual theoretical references, methodological decisions, development of the analysis process, reflections, learning and conclusions of the process. Similarly, a documentary review was carried out with the help of the following instruments: intervention projects, descriptive letters, group chronicles, supervision reports and operational programs. The validation of the information was accomplished by the judgment of experts.

As a result, the roles of the social worker were identified: social educator, sociocultural animator and social manager; in addition to the creation of virtual spaces for dissemination, participation and interaction with the population, and finally, the rigor of the methodology used.

In conclusion, the methodological process of community practice, integrated into five substantial stages, framed in the use of virtual media, contributed to the development of professional skills, responding to the context of the new reality.

Keywords: Systematization; Intervention; Virtual projects; Community.

Sumario: 1. Introducción, 2 Contexto de la experiencia, 2.1 Campo Práctico en Comunidades, 2.2 La práctica comunitaria en la modalidad virtual, 3. Referentes teóricos conceptuales, 4. Metodología, 4.1 Delimitación de la sistematización, 4.2 Colectivos participantes de la experiencia, 4.3 Técnicas y estrategias de recolección de información con fines de sistematización, 4.4 Plan de análisis de la información, 4.5 Procedimientos de validación, 5. Hallazgos 6. Conclusiones, 7. Referencias bibliográficas.
\end{abstract}




\section{Introducción}

Este texto tiene como objetivo exponer los aprendizajes profesionales de Trabajo social realizado en el Campo Práctico en Comunidades en el año 2021, en el marco de intervención a través de contextos virtuales, realizado por una universidad de nivel superior del estado de Yucatán, México. La sistematización se retoma de la etapa de intervención del proceso metodológico de Trabajo Social, que corresponde a la ejecución de proyectos sociales.

Basado en la propuesta de sistematización utilizada, se inicia con el apartado nombrado contexto de la experiencia, describiendo la naturaleza del Campo Práctico en Comunidades, el proceso previo a la práctica y las etapas que anteceden la intervención con los ajustes derivados de los protocolos sanitarios a consecuencia de la COVID-19; posteriormente se continúa con los referentes teóricos en donde se definen términos que aportan a la experiencia a sistematizar.

El siguiente apartado aborda las decisiones metodológicas: comprende la delimitación de la experiencia a sistematizar, los objetivos que la guían, los colectivos participantes, técnicas, instrumentos, plan de análisis y validación de la información. Se prosigue con las reflexiones y aprendizajes obtenidos, divididos en tres subtítulos que definen los roles y estrategias de intervención y proponen una estructura metodológica con ajustes a la modalidad virtual. Finalmente, se añaden las conclusiones destacando las reflexiones finales, retos y propuestas.

\section{Contexto de la experiencia}

\subsection{Campo Práctico en Comunidades}

La Licenciatura en Trabajo Social ${ }^{1}$ (LTS) de la Universidad Autónoma de Yucatán (UADY), realiza su intervención social comunitaria en la asignatura Campo Práctico en Comunidades como parte del plan de estudios 2015. Dicha práctica se lleva a cabo en el tercer semestre, ubicado en el nivel de formación inicial y eje disciplinar, que tiene como propósito que "el estudiante ejecute un proyecto de intervención relacionando la teoría y la práctica en una comunidad, respondiendo al diagnóstico situacional previamente elaborado, para su evaluación (...)" (Facultad de Enfermería de la Universidad Autónoma de Yucatán [FEUADY], 2015, p. 145).

El proceso metodológico del campo práctico se enmarca en cinco etapas: diagnóstico, programación, intervención, supervisión y evaluación las cuales se desarrollan en el segundo y tercer semestre del plan de estudios, y dirigidos a un mismo propósito, que el estudiante

1 La Facultad de Enfermería, UADY, en el año 2009 oferta la Licenciatura en Trabajo Social visualizando esta profesión prioritaria durante la intervención interdisciplinaria en el ámbito comunitario, misma que se vincula estrechamente con la salud del ser humano. 
Tzec-Puch, Ceh-Alvarado y González-Canul

adquiera competencias específicas para el quehacer del Trabajo Social en el ámbito comunitario, desde los niveles de intervención: caso, grupo y comunidad, es decir, se habla de una formación académica de doce meses, donde convergen diversas asignaturas enfocadas a la intervención comunitaria, teniendo dos momentos claves: el previo y el durante. A continuación, se representa este proceso en la Figura 1.

Figura 1. Proceso metodológico del Campo Práctico en Comunidades.

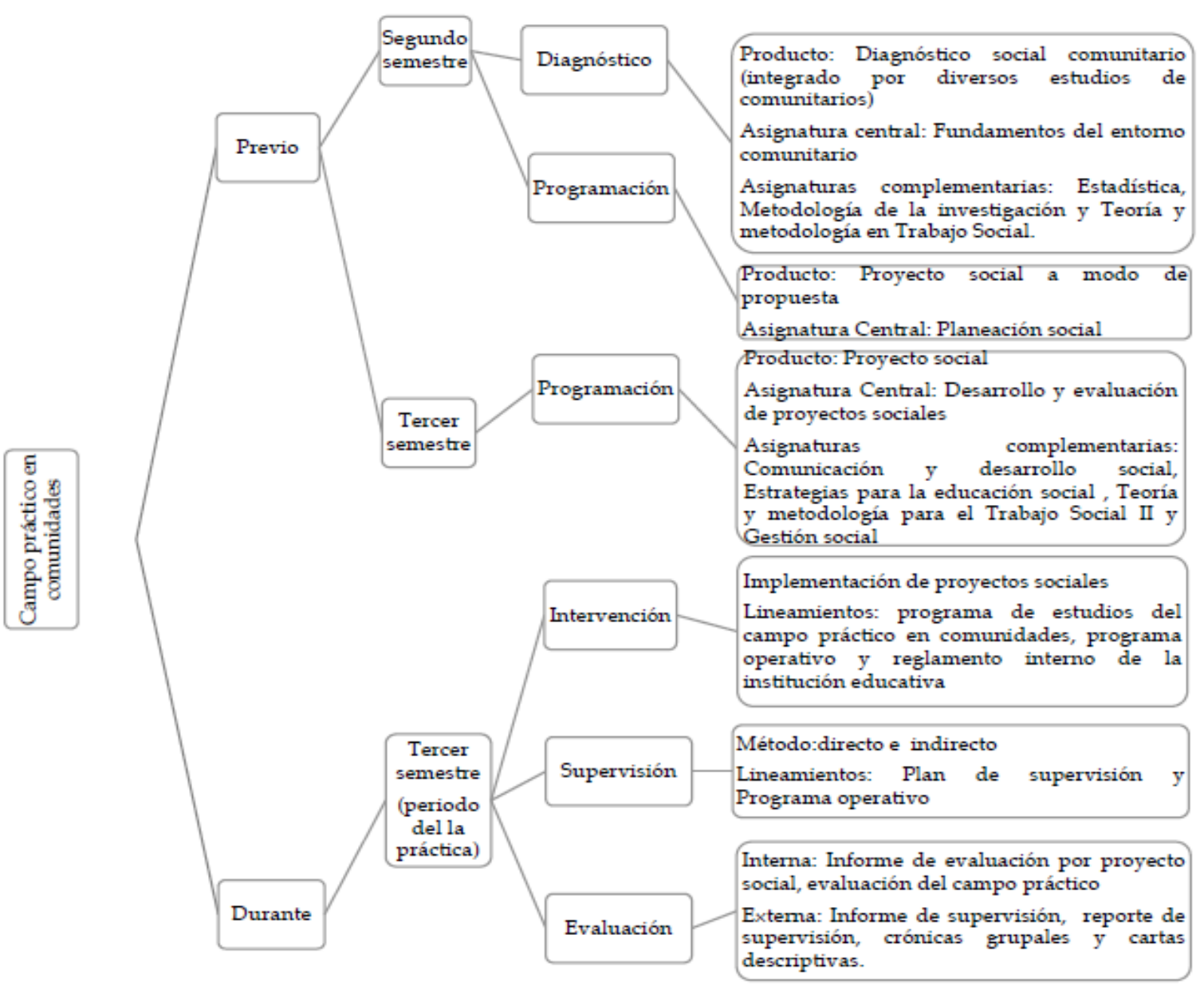

Fuente: elaboración propia a partir del plan de estudios de la LTS, 2021.

En la Figura 1, se presenta la primera etapa de la práctica ubicada en el segundo semestre, denominada diagnóstico, que tiene como propósito identificar las problemáticas y/o necesidades de una comunidad focalizada, a través de un conjunto de estudios comunitarios integrados en el producto académico nombrado diagnóstico social, correspondiente a la asignatura Fundamentos del entorno comunitario. Para la elaboración del producto académico contribuyen otras asignaturas, como son: Estadística, a través del análisis e interpretación de datos cuantitativos; Metodología de la investigación, con el 
Tzec-Puch, Ceh-Alvarado y González-Canul

análisis e interpretación de datos cualitativos; y Teoría y metodología en Trabajo Social, con la aplicación de las técnicas visita domiciliaria, entrevista y observación.

Continuando en el segundo semestre y con relación a la segunda etapa denominada programación, esta tiene como propósito establecer propuestas de intervención ante las problemáticas y / o necesidades detectadas en el diagnóstico social, es decir, en la asignatura Planeación social surge un proyecto preliminar que se concreta en el tercer semestre a través de la asignatura Desarrollo y evaluación de proyectos sociales, cuya competencia radica en la integración de elementos teóricos y metodológicos en la formulación y evaluación de proyectos sociales. Es importante señalar que contribuyen otras asignaturas como se explica en la Tabla 1.

Tabla 1. Contribución de asignaturas.

\begin{tabular}{ll}
\hline Asignatura & Actividad \\
\hline Comunicación y desarrollo & Elaboración de materiales de difusión para promocionar \\
social & los proyectos de intervención. \\
Estrategias para la & Elaboración de materiales didácticos para implementar \\
educación social & en la intervención. \\
Teoría y metodología para & Elaboración de un manual de técnicas y dinámicas \\
el Trabajo Social II & grupales para ejecutar con la población directa. \\
Gestión social & Elaboración de oficios de gestión para solicitar bienes \\
& materiales y/o de servicios prestados. \\
\hline
\end{tabular}

Fuente: elaboración propia.

En la Tabla 1, se identifica la integración de cuatro asignaturas que contribuyen a la programación de proyectos sociales, en este proceso se tiene como eje central el proyecto de intervención social. Asimismo, se enlistan las actividades que se generan en ellas, teniendo como funciones esenciales la difusión, la educación y la gestión social. El trabajo colaborativo de las asignaturas favorece la formación profesional del estudiante, centrado en el Trabajo Social comunitario en el nivel de intervención grupal.

Por otra parte, existe una tercera etapa denominada intervención ubicada en el tercer semestre, donde se implementa el Campo Práctico en Comunidades a través de la ejecución de los proyectos sociales, previamente trabajados en el segundo y tercer semestre, es decir, en la etapa de programación. La asignatura Campo Práctico en Comunidades tiene como competencia general que el estudiante "desarrolle un proyecto de intervención social en una comunidad aplicando los procesos de evaluación y sistematización que permitan atender 
Tzec-Puch, Ceh-Alvarado y González-Canul

las necesidades sociales con la participación de la población y en coordinación con las instituciones públicas y sociales" (Facultad de Enfermería de la Universidad Autónoma de Yucatán, 2015, p. 145).

Para llevar a cabo la práctica se determina un periodo de 20 días hábiles, teniendo como marco referencial el programa de estudios de la Licenciatura en Trabajo Social, el programa operativo del campo práctico en comunidades y el reglamento interno de la Facultad de Enfermería, documentos que rigen los lineamientos de la práctica.

Durante el periodo de práctica se identifica la etapa de supervisión, realizado por la coordinadora de los campos prácticos de la LTS, la coordinadora del campo práctico en comunidades y las supervisoras, cuya finalidad es acompañar y guiar al estudiante durante las actividades comunitarias. Para esta etapa los lineamientos que rigen son el programa operativo y el plan de supervisión.

Finalmente, durante el periodo de práctica se considera la última etapa del proceso conocida como evaluación, la cual tiene como objetivo monitorear de manera interna y externa el proyecto de intervención. Los documentos que guían el proceso interno son el informe de evaluación por proyecto social y evaluación del campo práctico; los que alinean el externo son el informe y reporte de supervisión, crónicas grupales, así como las cartas descriptivas.

\subsection{La práctica comunitaria en la modalidad virtual}

A partir de la contingencia sanitaria, consecuencia de la pandemia mundial de COVID-19, la virtualidad ha sido un elemento esencial en las intervenciones de índole social, económica, de salud, entre otras. Ante esta nueva realidad, la Comisión Económica para América Latina y el Caribe (CEPAL, 2020). asegura que la digitalización será un elemento principal

En Mérida, Yucatán, México, la contingencia sanitaria inició en el mes de marzo del año 2020, lo cual conllevó a una serie de normativas impuestas por el Gobierno del Estado, entre las que se incluyeron: suspensión de actividades escolares y eventos masivos, aislamiento social, entre otras medidas, mismos que se mantienen hasta la fecha actual (junio de 2021).

Por lo anterior, el proceso metodológico de la práctica se adaptó a la modalidad virtual, las etapas que se alinearon a esta fueron la programación, intervención, supervisión y evaluación, teniendo como excepción la etapa de diagnóstico, debido a que los estudios comunitarios se iniciaron de forma presencial pero no continuaron; ante esta situación, con 
los datos recabados y a partir de una revisión documental se concluyó con la etapa diagnóstica. A continuación, se explican las modificaciones realizadas:

\section{Diagnóstico}

Reconocida por Castro-Guzmán (2017) como diagnóstico situacional, menciona que en esta etapa se debe "identificar los principales problemas y necesidades sociales; ello con la finalidad de indagar la problemática social (...) caracterizar y ver la magnitud de las causas para su posible intervención" (p. 22). Esta etapa se llevó a cabo durante los primeros meses del segundo semestre de la LTS, en este se trabajó de manera presencial en una comunidad focalizada, elaborando estudios comunitarios: exploratorio, descriptivo, correlacional y explicativo, no obstante, este proceso no finalizó de manera presencial por las medidas de contingencia antes mencionadas y se tuvo que trabajar con los datos generados hasta marzo 2020 en modalidad virtual, cuyos resultados preliminares fueron: inseguridad, fauna nociva, falta de alumbrado público, drogadicción y alcoholismo.

Es importante resaltar que el plan de estudios de la LTS, 2015 está bajo el enfoque del modelo educativo para la formación integral, que incluye la plataforma "UADY virtual", denominado como "un sistema de educación en línea (modalidad mixta), la educación virtual (modalidad no presencial), para dar vida a un entorno virtual de aprendizaje" (Universidad Autónoma de Yucatán [UADY], 2021).

\section{Programación}

Entendida por Castro-Guzmán (2017, p. 14) como programación social, indica que en esta "se diseñan planes, programas y proyectos de intervención en atención a los problemas y necesidades sociales (...) bajo un enfoque teórico". En el mismo sentido y posterior a la elaboración del diagnóstico, en el tercer semestre, cursado en agosto-diciembre 2020, se analizaron las problemáticas y/o necesidades sociales de la comunidad para formular cuatro proyectos de intervención social en modalidad virtual con el fin de dar respuesta a los hallazgos. A pesar del esfuerzo por continuar trabajando con la población focalizada, no se tuvo éxito, ya que no toda la sociedad estaba preparada para adaptarse a los entornos virtuales, es decir, "en la práctica (...) el desarrollo tecnológico no ha resultado un proceso homogéneo y accesible, ni para todas las personas, ni en todos los contextos socioculturales" (Arriazu-Muñoz y Fernández-Pacheco Sáez, 2013. p. 150), por otra parte, la brecha digital deja de manifiesta un nuevo elemento de inequidad y la desigualdad, sobre todo en América Latina (CEPAL, 2020).

Aunado a ello, se estableció una estrategia durante el tercer semestre para validar la factibilidad de continuar con los proyectos considerando otra población, para ello se utilizó la metodología del marco lógico según Fundación Merced (2018) llevando a cabo el análisis 
Tzec-Puch, Ceh-Alvarado y González-Canul

de involucrados a actores claves y la técnica participativa diagnóstica árbol de problemas a través de entrevistas virtuales; lo anterior teniendo como población focalizada en un $50 \%$ a familiares y/o conocidos de los/las estudiantes. Finalmente, se analizaron los datos de las entrevistas y se formularon los proyectos virtuales de intervención que a continuación se explica en la siguiente etapa.

\section{Intervención}

Para Castro-Guzmán (2017) denominada como ejecución, declara que es "la realización de los proyectos" (p. 17). Continuando con la misma vertiente, durante el mes de enero y febrero del año 2021, se ejecutaron proyectos virtuales sincrónicos, es decir, Arriazu-Muñoz y Fernández-Pacheco Sáez (2013) mencionan que "la sincronía viene determinada por la confluencia en el espacio y tiempo de los interlocutores, independientemente de su contexto geográfico" (p. 150). Por consiguiente, los proyectos fueron dirigidos a la población de niños/as, adolescentes, adultos jóvenes y personas adultas mayores, a través de fechas y horarios establecidos para conectarse a las sesiones virtuales. Dichas sesiones se impartieron tres veces por semana con duración de 40 a 60 minutos aproximadamente. Las temáticas abordadas fueron: participación social; higiene personal y en las mascotas; adecuado uso del tiempo de ocio; y prevención del alcoholismo y drogadicción. En este periodo participaron 40 personas de Mérida y de otros países como lo son Bolivia y Perú.

Ante esta inclusión internacional, es importante destacar que la difusión de los proyectos se realizó a los habitantes de Mérida a través de redes sociales, pero se tuvo un mayor alcance, como lo indica Hütt-Herrera (2012)

\footnotetext{
las redes sociales y los espacios virtuales son mecanismos de interacción, simples canales de comunicación, pero con un potencial de alcance y de impacto mucho mayor que cualquiera de los otros medios tradicionales, y con capacidad de interacción y dinamismo, superiores a cualquiera de los otros medios. (p. 8)
}

Con relación a la elaboración de materiales de difusión, didácticos, manuales de técnicas, dinámicas grupales y oficios de gestión, estos productos se digitalizaron a través de diversas herramientas virtuales. Por consiguiente, se destaca la efectividad de desarrollo de competencias disciplinares y las experiencias de aprendizajes, debido a que las estrategias metodológicas virtuales favorecieron a los mecanismos y estilos de aprendizaje utilizando medios audiovisuales.

\section{Supervisión}

Para Castro-Guzmán (2017), esta etapa “hace referencia al trabajo de seguimiento y vigilancia oportuna en el desarrollo de las tareas y actividades" (p. 22). Esta etapa se realizó 
en el mismo periodo de la práctica (enero-febrero 2021), teniendo como documentos de referencia el programa operativo y el plan de supervisión de la práctica, ambos digitalizados. Se contó con la participación de la coordinadora del campo práctico en comunidades y cuatro supervisoras, tres de ellas docentes de la LTS y una pasante de la misma disciplina que se integró una semana antes de la conclusión de la práctica. Cabe señalar que, durante la supervisión cada docente guio un proyecto social, monitoreando las actividades realizadas para el alcance de los objetivos planificados.

La supervisión se efectuó todos los días de manera virtual a través de dos plataformas digitales (UADY virtual y Microsoft Teams), además se complementó con otros recursos como el WhatsApp y llamadas telefónicas, con el fin de tener una comunicación directa y continua.

Otro elemento importante de la supervisión fue la implementación semanal de talleres pedagógicos virtuales, es decir, espacios para expresar el desempeño de la práctica, compartir experiencias, implementar o reforzar temas académicos. En estos espacios se impartió el tema de diario de campo y se reforzó el tema de evaluación de proyectos, a su vez, se abordaron los retos y desafíos de la práctica en contextos virtuales, aprendizajes personales y profesionales, teniendo como resultado una mejora continua en la intervención y en el alcance de competencias disciplinares, genéricas y específicas.

\section{Evaluación}

En esta etapa "se realizan acciones que permiten comparar (...) las distintas etapas del proceso metodológico de intervención” (Castro-Guzmán, 2017, p. 22), para evaluar el proyecto social se consideraron los instrumentos declarados en el Programa Educativo de la Licenciatura en Trabajo Social y el programa operativo: criterios de evaluación de los proyectos sociales, informe de evaluación, crónica grupal, carta descriptiva e infografía. Para concluir, se destaca el logro de los objetivos planteados.

Para finalizar la explicación de la adaptación virtual del campo práctico, es relevante mencionar que durante las etapas del proceso de la práctica resaltaron las siguientes plataformas digitales: OneDrive, Google Drive, Google Classroom, Google Forms, Microsoft Teams, Zoom y Redes sociales como Facebook, Instagram, WhastApp, Podcast y Youtube. Las plataformas y medios digitales mencionados fueron consensuados con los beneficiarios del proyecto, considerando el contexto del participante, el acceso a la conectividad del internet, los dispositivos disponibles y el uso y manejo de estos. 


\section{Referentes teóricos conceptuales}

Como parte del marco teórico conceptual de la sistematización se incluyen los términos que son relevantes para la comprensión de la experiencia.

En primera instancia, se aborda la conceptualización de sistematización que es "el proceso participativo que permite ordenar las experiencias vividas; es decir, lo acontecido desde el diseño, elaboración, gestión, ejecución y evaluación de un proyecto" (CastroGuzmán, 2016, p. 9).

Bajo esta conceptualización, otros autores han contribuido al desarrollo teórico de la sistematización. En este sentido, Castañeda-Meneses (2015) plantea que la sistematización es "una conexión directa con su núcleo histórico fundacional organizado en torno a la intervención social. Es la posibilidad de atesorar sus estrategias, de fortalecer sus repertorios profesionales, de comunicar la valía de su trabajo cotidiano" (p.31).

Con base en estas definiciones planteadas, para fines de este texto se entenderá como sistematización el proceso permanente y acumulativo de creación de conocimientos que permite recabar y rescatar información relevante de un proyecto o intervención, a fin de retomar los datos y experiencias más significativas.

Por otra parte, la intervención comunitaria es entendida como "el conjunto de acciones destinadas a promover el desarrollo de una comunidad a través de la participación activa de esta en la transformación de su propia realidad" (Instituto Interamericano para el Desarrollo Social y Económico [INDES], 2000). Por tanto, pretende la capacitación y fortalecimiento de la comunidad, favoreciendo su autogestión para su transformación y la de su ambiente, dando a la comunidad capacidad de decisión y de acción. Por ende, debido a la escasa literatura del ámbito comunitario, se comprenderá como al espacio o ambiente donde se desarrolla un grupo de personas.

Por otro lado, Plant (2004) refiere las comunidades virtuales como "un grupo colectivo de entidades, individuos $u$ organizaciones que permanecen juntos temporalmente $o$ permanente a través de un medio electrónico para interaccionar recíprocamente en un espacio común de acuerdo con algún problema o interés" (Meirinhos y Osório, 2009, p. 47).

Continuando con la descripción de términos, el proyecto de intervención hace referencia "a un conjunto de actividades concretas, interrelacionadas y coordinadas entre sí, que se realizan con el fin de producir determinados bienes y servicios capaces de satisfacer necesidades o resolver problemas" (Ander-Egg y Aguilar-Idáñez, 2005, p. 10). 
Tzec-Puch, Ceh-Alvarado y González-Canul

Ahora bien, para efectos del estudio y considerando que a raíz de la pandemia surge este término, se definirá proyecto en modalidad virtual "al proceso de planificación de objetivos formativos para un período determinado, actividades y otros elementos necesarios para alcanzar dichos objetivos y desarrollar el proceso de enseñanza-aprendizaje a distancia" (Marciniak, 2017, p. 54).

Asimismo, como modalidad virtual al medio de comunicación donde las personas intercambian información mediante el uso de las Tecnologías de la Información y la Comunicación (TIC). Así como, medios digitales a los canales de comunicación a través de los cuales, se puede crear, observar, transformar y conservar la información en una gran variedad de dispositivos electrónicos digitales, como por ejemplo las páginas y sitios web.

Con respecto a la definición de nueva realidad, la cual tiene una escasa literatura, para este estudio se entenderá como aquellos cambios y adaptaciones en la interacción social, en conjunto con las diversas normas sanitarias que han surgido en la sociedad a partir de la pandemia mundial originada por la COVID-19.

Por otra parte, se comprenderá como entornos virtuales "el espacio o comunidad organizados con el propósito de lograr el aprendizaje, y que para que éste tenga lugar requiere ciertos componentes: una función pedagógica, la tecnología apropiada a la misma y el marco organizativo" (Salinas- Ibáñez, 2004, p. 33).

Finalmente, para este estudio se entenderá la intervención de Trabajo Social en tiempos de COVID-19 como todas aquellas acciones orientadas a la búsqueda de bienestar colectivo y social en el contexto de pandemia, mediante funciones de prevención y promoción a la salud; y el Trabajo Social comunitario en la nueva era digital a la integración de la tecnología en las competencias y estándares de su práctica para el diseño de estrategias que respondan a las necesidades de la comunidad.

\section{Metodología}

La sistematización es una estrategia de generación de conocimientos óptima a realizar cuando el análisis deriva de los aprendizajes de una práctica social (Castañeda-Meneses, 2015). Se escoge este proceso, puesto que lo descrito en el artículo corresponde a las vivencias del campo práctico en el ámbito comunitario durante la nueva realidad del COVID-19, mismo que conllevó a una reconfiguración del proceso de intervención social.

La recuperación de las experiencias que se realiza tiene como estructura base los componentes metodológicos que propone Castañeda-Meneses (2015), iniciando con el contexto de la experiencia, el marco teórico conceptual, marco metodológico, desarrollo del proceso de análisis, y, por último, las reflexiones, aprendizajes y conclusiones. 
Tzec-Puch, Ceh-Alvarado y González-Canul

Con respecto al marco metodológico, se centra en seis puntos a considerar: delimitar la sistematización según los objetivos, colectivos participantes, técnicas e instrumentos de recolección de información, plan de análisis y los procedimientos de validación.

Por lo anterior, se plantea la siguiente pregunta de estudio ¿Cuáles son los roles y las estrategias profesionales aplicadas por Trabajo Social en los proyectos sociales comunitarios en modalidad virtual?

\subsection{Delimitación de la sistematización}

La sistematización del presente documento consiste en recuperar los aprendizajes profesionales de la práctica social adquiridos durante el campo práctico en comunidades, que corresponde a la etapa de intervención del proceso metodológico de Trabajo Social, mediante su intervención en contextos virtuales durante el distanciamiento social causado por la COVID-19. Lo antes mencionado, se guía por dos propósitos principales:

- Definir los roles y las estrategias profesionales del trabajador social en la aplicación de proyectos sociales a través de medios digitales.

- Sistematizar el proceso metodológico comunitario de Trabajo Social en entornos virtuales para futuros referentes de aplicación práctica.

\subsection{Colectivos participantes de la experiencia}

Para la sistematización de la experiencia se consideraron distintos colectivos que tuvieron una relación directa durante la práctica, mismos que se enlistan a continuación mencionando las funciones sustanciales que realizaron.

- Coordinadora de la LTS. Verificar el proceso de cumplimiento del campo práctico.

- Coordinadora de los campos prácticos de la LTS. Supervisar el cumplimiento del programa operativo y realizar la evaluación del campo práctico.

- Coordinadora del campo práctico en comunidades. Realizar el programa operativo, establecer cronograma de actividades, talleres pedagógicos, monitoreo, seguimiento de la práctica y elaborar el informe de supervisión.

- Supervisoras del campo práctico. Monitorear el avance del proyecto mediante el seguimiento y revisión de los productos, observación participante durante la práctica, elaborar reportes de supervisión de manera semanal e informes al finalizar el periodo.

- Prestadora del servicio social de LTS. Colaborar en las funciones asignadas de la coordinadora de campo práctico. 
Tzec-Puch, Ceh-Alvarado y González-Canul

- Estudiantes del tercer semestre de la LTS. Ejecución de los proyectos sociales, elaborar materiales de educación social, sesiones virtuales con la población y gestión social.

- Población objetivo. Participantes de cada uno de los proyectos.

\subsection{Técnicas y estrategias de recolección de información con fines de sistematización}

Con respecto a las estrategias para la recopilación y rescates de las experiencias se utilizaron técnicas e instrumentos que facilitaron el análisis de los aprendizajes y la generación de conocimiento. La primera técnica fue la observación participante, ya que los colectivos que se incluyen en la sistematización estuvieron durante el proceso de práctica de manera constante en las siguientes actividades: talleres pedagógicos, sesiones de intervención con la población, creación de contenidos en redes sociales, evaluación de la práctica.

Continuando con las técnicas para sistematizar, se empleó la revisión documental, mediante la cual se contemplaron diversos instrumentos que han sido resultado de la intervención comunitaria, o bien, han fungido como fuentes de verificación para indicadores de resultados. Estos se dividen en dos grupos, los relacionados con el proyecto e intervención directa; y los que establecen los procesos de práctica tales como: programación, intervención, supervisión y evaluación.

A. Relacionados con la intervención directa:

- Proyectos de intervención. Se rescatan el objetivo, metas, actividades directas e indirectas con la población, técnicas, metodologías y aspectos fundamentales de la intervención.

- Cartas descriptivas. Contienen la programación de cada una de las sesiones directas con la población, describe las técnicas, temas y evaluación.

- Crónicas grupales. Redacta los acontecimientos más relevantes de la sesión realizada y su interpretación, en conjunto con la evaluación de los objetivos y metas.

- Informe de evaluación del proyecto. Presenta los resultados obtenidos durante la aplicación del proyecto social, describiendo el cumplimiento de sus objetivos, metas, indicadores y logros de carácter cualitativos o cuantitativos. 
B. Relacionados con los procesos de práctica:

- Programa operativo. Contiene la competencia, productos de aprendizaje, estrategias de evaluación, distribución de estudiantes y cronograma de actividades.

- Reportes de supervisión. Registran las actividades realizadas por los estudiantes y las funciones directas e indirectas desempeñadas durante la práctica.

- Informes de supervisión. Dan a conocer las actividades que se realizan durante el campo práctico, en donde se describen el conjunto de funciones aplicadas, aprendizajes, retos y observaciones a partir de la intervención comunitaria.

Como última técnica de recolección de datos, se llevaron a cabo reuniones de trabajo en las cuales se compartieron experiencias y opiniones que conllevaron a una validación de juicio por experticia de las personas participantes; éstas consistieron en compartir puntos de vista de la sistematización, realizar la verificación de fuentes de información para la obtener experiencias, aprendizajes y conocimientos empíricos.

\subsection{Plan de análisis de la información}

El plan de análisis de la información elegido fue el de contenido, debido a que se revisaron documentos elaborados donde se recuperaron las vivencias, experiencias y aprendizajes de la práctica, desde la técnica de observación participante y revisión documental. Para el desarrollo de la sistematización se tomaron como referentes dimensiones vinculadas con los objetivos planteados, mismos que se presentan en la Tabla 2:

Tabla 2. Categorías de análisis.

\begin{tabular}{|c|c|c|c|}
\hline $\begin{array}{l}\text { Categoría de } \\
\text { análisis }\end{array}$ & Definición teórica & Definición analítica & Instrumentos de análisis \\
\hline $\begin{array}{l}\text { Rol } \\
\text { profesional }\end{array}$ & $\begin{array}{l}\text { Se visualiza como el papel que } \\
\text { ejerce ante diversas situaciones a } \\
\text { través de las funciones que } \\
\text { desarrolla el Trabajo Social, } \\
\text { realizadas de forma regular, } \\
\text { sistemática y sujeta a los contextos } \\
\text { con el fin de alcanzar los objetivos } \\
\text { profesionales (Kisnerman, 2005). }\end{array}$ & $\begin{array}{l}\text { Análisis y reflexiones } \\
\text { de las funciones } \\
\text { aplicadas. Ventajas que } \\
\text { aporta la modalidad } \\
\text { virtual para el } \\
\text { desarrollo de } \\
\text { funciones. }\end{array}$ & $\begin{array}{l}\text { Proyecto de intervención. } \\
\text { Crónicas grupales. } \\
\text { Programa operativo. } \\
\text { Reporte e informe de } \\
\text { supervisión. }\end{array}$ \\
\hline $\begin{array}{l}\text { Estrategias de } \\
\text { intervención }\end{array}$ & $\begin{array}{l}\text { Conjunto de recursos utilizados } \\
\text { con el propósito de desplegar } \\
\text { tareas en un determinado espacio } \\
\text { social y cultural (Rodríguez citado } \\
\text { en Barreno-Salinas, Astudillo- } \\
\text { Cobos y Barreno-Salinas, 2018). }\end{array}$ & $\begin{array}{l}\text { Análisis de las recursos } \\
\text { y tareas aplicadas en el } \\
\text { Campo Práctico desde } \\
\text { un marco virtual. }\end{array}$ & $\begin{array}{l}\text { Proyecto de intervención. } \\
\text { Cartas descriptivas. } \\
\text { Crónicas grupales. } \\
\text { Informe de supervisión. }\end{array}$ \\
\hline
\end{tabular}

Fuente: elaboración propia. 


\subsection{Procedimientos de validación}

Con el fin de validar los datos obtenidos y que sean objetivos, consensuados y generen una opinión informada que respalde o ajuste los resultados de la experiencia, se llevó a cabo un juicio de experticia de personas participantes en el proceso, siendo tres las involucradas: la coordinadora de prácticas, una supervisora y la prestadora de servicio social. Cada una de ellas estuvo involucrada durante el proceso de prácticas, elaboración/revisión de al menos un instrumento, $\mathrm{y}$, además, participaron en todas las técnicas de recolección de datos.

Asimismo, como complemento a la validación de la información recolectada se retomó la triangulación de fuentes, puesto que durante la técnica de revisión documental se consideraron instrumentos elaborados por distintos colectivos. En conjunto con lo anterior, es preciso mencionar que el campo práctico se encuentra adscrito al programa de buenas prácticas institucionales de calidad, por lo cual cada uno de los instrumentos cuentan con formatos, instrumentos y documentos revisados y aprobados por el sistema de calidad.

\section{Hallazgos}

La intervención social a través de proyectos comunitarios, bajo el marco institucional de la FEUADY y en el contexto de la nueva realidad, requirió de un repensar en el actuar profesional del Trabajo Social, la cual se reflejó en la creación de ámbitos comunitarios que facilitaran la participación de los grupos focalizados. Durante la experiencia descrita, se identificaron los roles profesionales y las estrategias de intervención que permiten replantear el proceso metodológico aplicado al Campo Práctico en Comunidades desde entornos virtuales.

\subsection{Roles profesiones de Trabajo Social en la aplicación de proyectos virtuales}

Para iniciar, el Trabajo Social ejerció diversas funciones de forma regular y sistemática en el contexto virtual con los sujetos participantes en cada uno de los proyectos antes mencionados, éstas fueron elementales para favorecer la participación de la población y que se cumplieran los objetivos establecidos. Los roles profesionales que destacaron durante la etapa de intervención fueron tres: educador social, animador sociocultural y gestor social. En los siguientes párrafos se describe cada uno de ellos con sus respectivas implicaciones en la práctica.

Uno de los primeros roles efectuados fue el de gestor social, mediante la toma de decisiones para la creación de vínculos y acciones conjuntas entre instituciones públicas o profesionales con la comunidad focalizada; así como la obtención de bienes o servicios que fueron de utilidad en la ejecución del proyecto de intervención. 
Para efectuar los proyectos sociales, la gestión inició con la obtención y creación de los distintos espacios virtuales de difusión, participación e interacción, los principales utilizados fueron: redes sociales como WhatsApp, Facebook, Instagram, TikTok; plataformas de videollamadas como Google Meet y Zoom, lo cual incluyó obtener las cuentas premium que facilitaran la intervención grupal con la población objetivo.

La gestión y uso de redes sociales virtuales permite reflexionar que estos espacios fungen como un medio de difusión de amplio alcance, que logra focalizar la atención de la población objetivo de los proyectos; el uso de medios digitales como medio de promoción tiene como ventajas: ser gratuitos, tener una interacción en tiempo real y a largo plazo, así como tener un alcance estatal, nacional e incluso internacional.

La elección de medios digitales y plataformas para la interacción proviene del análisis de una serie de características que se fundamentan en la dinámica de grupos, teniendo como criterios principales lo siguiente: tamaño, madurez y objetivo del grupo. Así entonces, a pesar de que la gestión fue a través de medios tecnológicos, se identifica la necesidad de una toma de decisiones fundamentada en los principios teóricos y disciplinares de Trabajo Social con grupos.

Siguiendo con la misma línea de análisis, la gestión social también se realizó por medio de la vinculación con distintos profesionales de instituciones públicas o que ejercen servicios privados, los cuales participaron como ponentes y expertos en algunas temáticas seleccionadas por cada proyecto según sus actividades programadas, siendo así un complemento de sus intervenciones.

De acuerdo con las experiencias redactadas, se reconoce que el rol de gestor social mantiene la toma de decisiones fundamentada y sistemática de la elección de recursos humanos, técnicos y materiales, afirmando que exige a la profesión un conocimiento amplio de herramientas tecnológicas, actualización constante y rigor metodológico ante las acciones realizadas.

Otro de los roles principales en la práctica fue el de educador social, en el cual se implementaron estrategias de educación y comunicación social con los grupos de población, con el propósito de lograr los objetivos propuestos en el proyecto de intervención social. Al ser una práctica virtual los trabajadores sociales en formación se enfocaron en emplear las herramientas y novedades que ofrecen estos espacios y que, al mismo tiempo, se adapten a su población objetivo.

Asimismo, es preciso resaltar que este rol conllevó a múltiples actividades las cuales incluyeron investigaciones documentales, elaboración de material didáctico, creación de 
Tzec-Puch, Ceh-Alvarado y González-Canul

instrumentos de evaluación y ajuste de técnicas grupales a la modalidad virtual. La implicación de este segundo rol se logró cumplir en su totalidad durante la práctica con ajustes e innovaciones que ofrece la intervención en línea, tales como mayor representación visual, uso de vídeos, proyecciones, interacción directa con el contexto, por señalar algunas.

Como aprendizajes obtenidos, la educación social es una función que se adaptó con facilidad al proyecto, ya que la escuela virtual proporcionó herramientas y habilidades digitales para la elaboración de materiales didácticos, creación de espacios y una programación virtual. Además, proporcionó la creación de contenidos digitales con el fin de reforzar la enseñanza mediante el uso de diferentes plataformas como son: videos en YouTube, podcasts, blogs.

Los hallazgos de esta sistematización demuestran la importancia del uso de los medios digitales y herramientas tecnológicas en el ejercicio profesional del trabajador social, en especial en la educación social, al facilitar el desarrollo del campo práctico en comunidades y, por ende, la ejecución de los proyectos sociales los cuales respondieron a las necesidades de la población. Aunado a ello, se encontraron similitudes en los resultados obtenidos de la investigación de los autores Cedeño-Rengifo, Loor-Lino y Vélez-Santana (2019) las cuales señalan que "el Trabajo Social en su ejercicio profesional utiliza la tecnología como una herramienta que permite fortalecer su intervención, apoyándose en aplicaciones y programas que sean de gran aporte" (p. 11).

Por último, se cumplió el rol de animador sociocultural, puesto que se produjeron procesos de sensibilización y motivación para que la comunidad asuma protagonismo mediante su participación, con el fin de satisfacer sus necesidades en el ámbito individual, familiar y comunitario. Este rol fue esencial durante la intervención y el logro de la competencia en el campo práctico, pero también para el logro de cada uno de los objetivos, metas e indicadores de los proyectos sociales efectuados; consistió en tres implicaciones principales: la creación de vínculos de confianza entre facilitadores-usuarios, creación de dinámicas participativas durante las sesiones, así como los resultados obtenidos al finalizar las intervenciones.

Recopilando el sentir de los sujetos participantes en la práctica, se enfatiza en que la comunicación efectiva entre trabajador social-usuario, no está condicionado al medio presencial o al uso de las herramientas tecnológicas, depende de las habilidades y actitudes profesionales que se tengan, desde el rapport, escucha activa, empatía, respeto, por mencionar algunas.

Respecto a los roles que se desarrollaron durante la ejecución de proyectos sociales se identifican los tradicionales de la profesión, siendo estos: gestor, animador y educador, sin embargo, se aplicaron estrategias distintas para la intervención en modalidad virtual, las 
Tzec-Puch, Ceh-Alvarado y González-Canul

cuales coinciden con las que señala Nomen (2021), puesto que se generaron nuevas aproximaciones a los usuarios desde las nuevas tecnologías, así como mediación con instituciones y usuarios.

\subsection{Estrategias de intervención en proyectos virtuales}

Continuando con la sistematización de la práctica, se describen las estrategias de intervención aplicadas por el trabajador social en el ámbito comunitario, las cuales según Rodríguez (2010) hacen referencia al conjunto de recursos utilizados con el propósito de desplegar tareas en un determinado espacio social y socio-cultural (Barreno-Salinas, Astudillo-Cobos y Barreno-Salinas, 2018), en este caso, durante el tiempo de confinamiento social, los equipos se enfocaron a la creación e interacción en espacios virtuales de acuerdo con las características de la población.

Una de las primeras estrategias fue el emplear los medios digitales como canales de comunicación para comenzar a crear comunidades virtuales, utilizando aquellas redes sociales que cumplían los siguientes criterios con la población: accesibilidad, conocimientos de su manejo y cercanía de la comunicación. Es importante señalar, que además de la intervención directa, se adaptaron las técnicas e instrumentos comunitarios de forma digital, así como procesos de la práctica como el encuadre, talleres pedagógicos, supervisión y cierre mediante videollamadas y chat del Microsoft Teams y Zoom.

El utilizar los medios digitales para la intervención aplicó una de las bondades que señaló Miranda-Ruche (2016), implicando que la “capacidad de organización y difusión que permite la estructura tecnológica facilita el encuentro entre personas y grupos con intereses comunes que desean relacionarse" (p.4). En este sentido, durante la práctica, se facilitó la interacción entre docentes-estudiantes, al mismo tiempo que la participación de población de distintos lugares geográficos con nivel estatal, nacional e internacional en los proyectos sociales.

A pesar de ser un medio virtual de intervención, se aplicaron las estrategias de técnicas grupales que cumplieron con los propósitos establecidos y fueron de gran apoyo para la ejecución del proyecto, entre las aplicadas se encuentran: de animación, expositivas, contenido temático, evaluación y retroalimentación.

Por lo tanto, el desarrollo del campo practico virtual fue una creación novedosa, al permitir una visión más holística, facilitar el acceso a ideas, experiencias y perspectivas tanto racionales como emotivas y generar la participación en la población objetivo. Asimismo, fue un espacio virtual que se utilizó para promocionar información y realizar actividades que demandó el campo práctico, por lo que se necesitó del apoyo de herramientas digitales para el cumplimiento de los objetivos y metas establecidas en la práctica. 
Tzec-Puch, Ceh-Alvarado y González-Canul

Como parte de los aprendizajes obtenidos, se cumplieron las habilidades del perfil del trabajador social, siendo estas las imprescindibles para la práctica virtual: (a) rapport, facilitó la confianza entre la población y facilitadores, propiciando una participación en cada una de las sesiones; (b) adaptación, se adecuaron las técnicas y materiales didácticos según el contexto de intervención, c) creatividad, se proporcionó una respuesta pronta ante las situaciones imprevistas.

Continuando con las reflexiones, fueron evidentes en los entornos virtuales las siguientes habilidades de educación social: (a) inducción, promovió la motivación de los participantes, y la preparación de las sesiones, (b) comunicación de tipo asertiva, (c) distintos tipos de interacción de la educación, siendo estos facilitador-participante y participante-participante, los cuales permitieron la interacción de conocimientos empíricos para la educación social, así como ejemplos apegados a la realidad de los participantes, acorde a su edad y contexto.

Dentro de las estrategias se visualizaron diversos modelos de comunicación y aprendizaje en cada uno de los proyectos de intervención, que favorecieron la intervención en el ámbito comunitario, adaptando las necesidades a los medios digitales. Entre los que cabe destacar: comunicación para el cambio de comportamiento, educación lúdica y el aprendizaje social.

\subsection{Proceso metodológico en la elaboración de proyectos sociales en entornos virtuales}

Para comenzar, es preciso señalar que el tipo de comunidad de intervención fue de tipo virtual, la cual desde años anteriores se ha identificado como un contexto emergente, debido al constante desarrollo tecnológico, y que, además, se rige por "las dinámicas de cambio que afectan a la noción de comunidad, y a las posibilidades de intervención que pueden surgir de los nuevos escenarios virtuales" (Miranda-Ruche, 2016, p. 3). En esta práctica, la dinámica de cambio provino de una pandemia mundial por la COVID-19 que tuvo como consecuencia la implementación de protocolos sanitarios para la intervención y los procesos de formación académicos.

Reflexionando acerca de las estrategias y los roles aplicados durante el proceso de intervención se identifica que se cumplió con el rigor metodológico que establece en cada uno de los documentos y metodologías que orientan la práctica, procurando el cumplimiento de las funciones, técnicas e instrumento desde las etapas de planeación, programación, intervención y evaluación. No obstante, por el hecho de estar inmersos en ámbitos comunitarios virtuales se concuerda con los autores Caravaca-Llamas (2014) y Casas-Ratia (2020) quienes hacen referencia a considerar replantear su actuación, práctica profesional, metodologías, modelos y estrategias de intervención, en el marco virtual, es 
Tzec-Puch, Ceh-Alvarado y González-Canul

decir, con el uso estratégico de las tecnologías. Lo anterior se relaciona con la práctica, ya que a partir de esta se identificaron diferentes estrategias, recursos y competencias que se aumentan y exige esta modalidad de trabajo.

Teniendo como referencia: las funciones, técnicas e instrumentos adaptados a escenarios virtuales, así como la relevancia de enfatizar estrategias durante el proceso de intervención, se propone un ajuste a la metodología en el ámbito comunitario en medios digitales, con fundamento en las necesidades, roles desarrollados y modificados; siendo así una base para futuras aplicaciones y consolidar un foco emergente de Trabajo Social en la virtualidad.

La sistematización de la experiencia dentro del contexto de la práctica identifica elementos importantes de retomar en cuanto a la intervención desde la modalidad virtual, sus roles, estrategias y el fundamento de su rigor metodológico. Así entonces, la síntesis metodológica del reajuste de la intervención que se propone se presenta en la Figura 2:

Figura 2. Metodología de Trabajo Social adaptada en la modalidad virtual

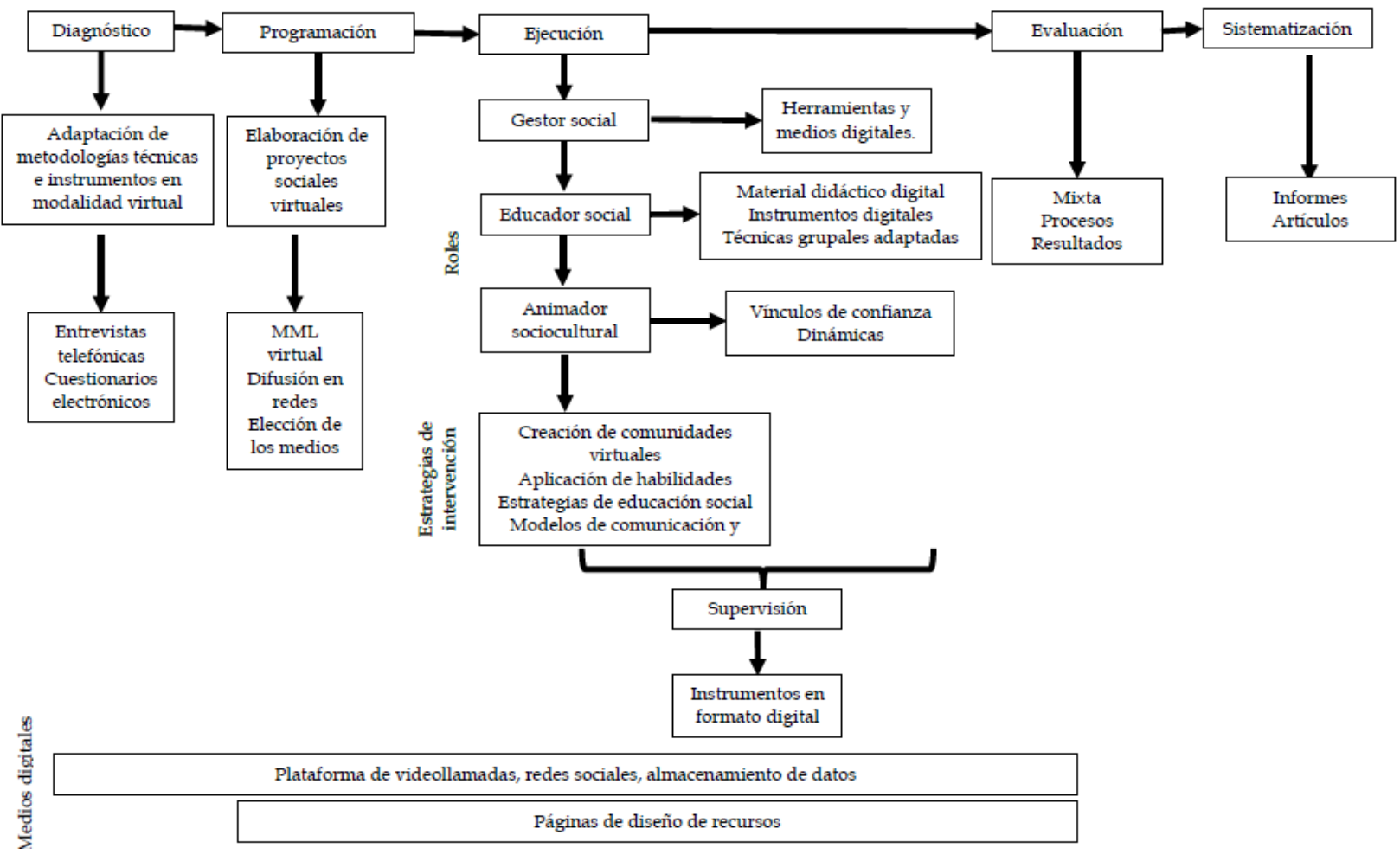

Fuente: elaboración propia. 
Tzec-Puch, Ceh-Alvarado y González-Canul

Como primera etapa se elabora el diagnóstico, con la finalidad de identificar las problemáticas y/o necesidades de una comunidad objetivo mediante la elaboración de estudios comunitarios; por ende, se deberá adaptar la metodología, técnicas e instrumentos en modalidad virtual, como lo son el uso de entrevistas telefónicas y cuestionarios digitales.

Asimismo, la programación, que corresponde a la elaboración de proyectos sociales virtuales, será de acuerdo con los hallazgos encontrados previamente en la primera etapa, los marcos metodológicos y el contacto con la comunidad puede llevarse a cabo mediante medios virtuales, considerando la elección de éstos y la accesibilidad de los sujetos que van a participar; por ende, en esta modalidad de intervención, se resalta como alternativa la difusión de los proyectos a través de redes sociales, así como la elección de medios digitales para todo el proceso, desde elaborar material didáctico hasta sesiones grupales.

Durante la etapa de intervención, que consiste en la aplicación de un proyecto de intervención, se desarrollan tres funciones principales: gestor social en el uso de herramientas y medios digitales; educador social en la aplicación de materiales didácticos e instrumentos digitales y técnicas grupales adaptadas; y animador sociocultural mediante los vínculos de confianza y dinámicas participativas. De igual modo, es imprescindible generar diversas estrategias de intervención como las comunidades virtuales, aplicación de habilidades, estrategias de educación social, modelos de comunicación y aprendizajes adaptados a la modalidad virtual.

La etapa de supervisión durante un proceso virtual requiere aplicarse desde el inicio de la intervención hasta la evaluación, misma a efectuar de forma directa e indirecta; la primera relacionada con el aprovechamiento del estudiante, desarrollo de habilidades, aplicación de proyectos sociales, valoración de relaciones humanas y actitudinales que permiten efectuar los roles profesionales; la segunda con relación a los instrumentos de evaluación de los productos de aprendizaje de la práctica, el reporte e informe de supervisión, las cuales se digitalizaron.

Se prosigue, con la evaluación enfocada en dos partes fundamentales, de proceso y de resultados, la primera debe de ser efectuada con los colectivos participantes de la institución, puesto que contribuye a que aquellas situaciones que tengan áreas de oportunidad o sean ineficaces puedan remediarse durante la práctica, la segunda, estará enfocada a la población participante presentando una valoración del cumplimiento de las metas y objetivos, así como a los colectivos de trabajo social analizando el logro de las competencias señaladas y propósitos establecidos. La etapa de evaluación puede emplear las plataformas de videollamadas, redes sociales, almacenamiento de datos y páginas de diseño de recursos. 
Tzec-Puch, Ceh-Alvarado y González-Canul

Finalmente, el proceso metodológico en nuevos contextos virtuales y emergentes requiere de manera indispensable contar con una etapa de sistematización, en la cual se elaboren informes de los resultados obtenidos desde una visión crítica y objetiva, además de, artículos que permitan difundir los procesos aplicados, generando así conocimiento situacional, empírico y disciplinar.

\section{Conclusiones}

Las medidas sanitarias, consecuencia de la COVID-19, implicaron el establecimiento de nuevas alternativas para la intervención social en el ámbito comunitario, mismo que conllevó a repensar y adecuar la ejecución de proyectos sociales. La experiencia de intervenir por primera vez en modalidad virtual y de adaptar los elementos teóricos y procesos metodológicos de la práctica en esta modalidad, trajo consigo retos, pero también oportunidades para la profesión.

Con relación a los retos profesionales, se centran en los medios, herramientas y estrategias digitales. El primero se presentó al elegir las plataformas digitales para ejecutar los proyectos sociales, debido a la diversidad y acceso a las mismas, es decir, no todas las personas tenían el conocimiento de éstas, ni los recursos técnicos. Por lo anterior, se tuvo que elegir las plataformas más comunes y utilizadas por la población beneficiada.

Otro reto fue la conectividad a internet por parte de la población y los estudiantes, debido a que en ocasiones se presentaban dificultades que implicaban cambios en la dinámica de trabajo. La nueva realidad en el Estado implicó que (en su gran mayoría) las actividades laborales y escolares se realizaran desde el hogar, por ende, la carga de actividades cotidianas y laborales fueron factores externos que condicionaban la participación de la población beneficiada.

A partir de la experiencia de intervención, se identifican las fortalezas de la práctica que radican en la adaptabilidad del proceso en entornos virtuales, teniendo así diversas herramientas tecnológicas que coadyuvaron al desarrollo, el cual propició que la comunidad de aprendizaje accediera fácilmente a éstas.

Si bien el contexto de intervención fue a través de medios digitales, se encontraron nuevas oportunidades para la profesión de Trabajo Social entre las cuales se mencionan las siguientes: adaptar los procesos teóricos metodológicos del Trabajo Social comunitario a la modalidad virtual, es decir, realizar nuevas formas de la animación sociocultural y promoción social; visualizar los entornos virtuales como un modo de tener mayor alcance local, estatal e internacional, debido a que durante la práctica participaron personas de otras localidades y países; por último, capacitar a los responsables de los proyectos sociales acerca 
de las nuevas plataformas y medios digitales que respondan a las necesidades actuales de intervención social.

A manera de reflexión, es posible afirmar que a través de las estrategias aplicadas se alcanzó la competencia de la práctica y se logró conformar una comunidad en un ambiente virtual, mediante la cual se intervino en distintas problemáticas. De tal manera que se aplicó el perfil profesional de Trabajo Social Comunitario a través de las diversas funciones mencionadas, desarrollando habilidades y actitudes que demandan este ámbito.

Como conclusión, se establece que el trabajador social es un profesional que se encuentra sujeto a las nuevas realidades que son consecuencia de los acontecimientos dentro del contexto social donde está inmerso, tal como la COVID-19, la cual ha traído nuevas formas de interactuar y de intervenir en la atención de necesidades sociales; por tal motivo, requiere adecuar a sus funciones, acciones y su proceso metodológico a la virtualidad.

\section{Referencias bibliográficas}

Ander-Egg, E., y Aguilar-Idáñez, M. (2005). Cómo elaborar un proyecto. Guía para diseñar proyectos sociales y culturales. Argentina. Lumen/Hvmanitas.

Arriazu-Muñoz, R., y Fernández-Pacheco Sáez, J. L. (2013). Internet en el ámbito del Trabajo Social: formas emergentes de participación e intervención sociocomunitaria. Cuadernos de Trabajo Social, 26(1), $\quad$ 149-158. doi: 10.5209/rev_CUTS.2013.v26.n1.41665

Barreno-Salinas, Z., Astudillo-Cobos, A., y Barreno-Salinas, M. M. (2018). Hacia una estrategia de intervención en la comunidad: referentes teóricos metodológicos. Revista Conrado, 14(62), 258-265. Recuperado de https:/ / conrado.ucf.edu.cu/index.php/conrado/article/view/715.

Caravaca-Llamas, C. (2014). Trabajo social on-line. El futuro de la intervención social. Pensamiento al margen: revista digital sobre las ideas políticas, (1), 1-17. Recuperado de https://www.pensamientoalmargen.com/1/trabajadora_social_de_redes_visto.pdf.

Casas-Ratia, C. G. (agosto, 2020). Redes sociales, familias y trabajo social: un reto o una estrategia de intervención ante la nueva cotidianidad. En Foro Nacional de Trabajo Social 2020. Retos, estrategias y acciones para la intervención social ante la nueva cotidianidad: Aportes desde Trabajo Social. Universidad Nacional Autónoma de México. Escuela Nacional de Trabajo Social, Ciudad de México, México. Recuperado de http://www.trabajosocial.unam.mx/comunicados/2020/agosto/foro/Mesa_5_Car men_Casas_Ratia.pdf.

Castañeda-Meneses, P. (2015). Sistematización y generación de conocimientos en Trabajo Social. Aportes metodológicos a la formación profesional. Alternativas. Cuadernos de Trabajo Social, (22), 23-32. doi: 10.14198/ALTERN2015.22.02. 
Tzec-Puch, Ceh-Alvarado y González-Canul

Castro-Guzmán, M. (2016). Sistematización en Trabajo Social: un proceso de construcción del conocimiento entre la práctica y la teoría. México: UADY.

Castro-Guzmán, M. (2017). Metodología para la investigación e intervención en Trabajo Social. En M. Castro-Guzmán, G., C. Reyna-Tejada, T., y J. Méndez-Cano, C. (Eds.), Metodología de Intervención en Trabajo Social (pp. 13-23). México: Universidad Autónoma de Yucatán. Universidad Nacional Autónoma de México, Academia Nacional de Investigación en Trabajo Social.

Cedeño-Rengifo, Y., Loor-Lino, L., y Vélez-Santana, A. (2019). El trabajador social y el uso de la tecnología como una herramienta útil para el ejercicio profesional. Revista Caribeña de Ciencias sociales, 2(18), 1-54. Recuperado de https:/ / www.eumed.net/rev/caribe/2019/12/trabajador-social-tecnologia.pdf.

CEPAL. (2020). La matriz de la desigualdad social en América Latina. Santiago, Chile: Cepal. Recuperado de https:/ / repositorio.cepal.org/bitstream/handle/11362/40668/4/S1600946_es.pdf

Facultad de Enfermería de la Universidad Autónoma de Yucatán [FEUADY]. (2015). Plan de estudios de la Licenciatura en Trabajo Social. México: UADY.

Fundación Merced. (2018). Diseño de proyectos sociales con enfoque de marco lógico. En D. Bolívar y A. Tapia, Manual Joven A.C. (pp. 33-60). México: SEDESOL, IMJUVE, JOVEN AC, FUNDACIÓN MERCED. Recuperado de https:/ / fundacionmerced.org.mx/wpcontent/uploads/2020/03/Fundacion-Merced-Manual-Joven-AC-2018-alta.pdf.

Hütt-Herrera, H. (2012). Las Redes Sociales: Una Nueva Herramienta de Difusión. Reflexiones, 91(2), 121-128. Recuperado de https:/ / www.redalyc.org/articulo.oa?id=72923962008.

Instituto Interamericano para el Desarrollo Social y Económico [INDES]. (2000). Diseño y gerencia de políticas y programas sociales. Recuperado de https://www.fundacionhenrydunant.org/images/stories/biblioteca/PoliticasPublicas/modelo\%20de\%20formacion\%20de\%20politicas\%20y\%20programas\%20so ciales \%20carlos\%20gerardo\%20molina.pdf

Kisnerman, N. (2005). Pensar el Trabajo Social. Buenos Aires, Argentina: LUMEN.

Marciniak, R. (2017). Propuesta metodológica para el diseño del proyecto de curso virtual: aplicación piloto. Rev Apert, 9(2), 65-80. doi: 10.32870/ap.v9n2.991.

Meirinhos, M., y Osório, A. (2009). Las comunidades virtuales de aprendizaje: el papel central de la colaboración. Pixel-Bit. Revista de Medios y Educación, (35), 45-60. Recuperado de https:/ / www.redalyc.org/pdf/368/36812381004.pdf.

Miranda-Ruche, X. (2016). La comunidad virtual como espacio de intervención para el Trabajo Social. elementos de análisis desde el ámbito de la salud mental. En D. Carbonero, E. Raya, N. Caparros y C. Gimeno, C. (Coords.), (2016). Respuestas transdisciplinares en una sociedad global. Aportaciones desde el Trabajo Social (pp. 1-15). Logroño: Universidad de La Rioja.

Nomen, L. (2021). La nueva normalidad y los futuros escenarios en Trabajo Social. Itinerarios de Trabajo Social, (1), 55-61. doi: 10.1344/its.v0i1.32432. 
Plant, R. (2004). Online communities. Technology in Society, 26(1) 51-65. Recuperado de http:/ / moya.bus.miami.edu/ rplant/papers/TinS.pdf.

Rodríguez, M. L. (2010). Estrategias de intervención. Algunos aspectos metodológicos y epistemológicos [Mensaje en un blog]. Recuperado de https:/ / metodologiasdelainvestigacion.wordpress.com/author/astrolabiodelsur/.

Salinas-Ibáñez, J. (2004). Cambios metodológicos con las TIC: estrategias didácticas y entornos virtuales de enseñanza-aprendizaje. Bordón. Revista de Pedagogía, 56(3-4) 469481. Recuperado de https:/ / dialnet.unirioja.es/ servlet/articulo?codigo=1291863.

Universidad Autónoma de Yucatán [UADY]. (2021). ¿Qué es UADY Virtual? Recuperado de https://www.uady.mx/uady-virtual. 


\section{OTROS ARTÍCULOS DE PROSPECTIVA No. 33}

\section{EDITORIAL}

El Paro Nacional en Colombia 2021: explosión social entre dinámicas estructurales y de coyuntura. Relevancia de la acción política y del diálogo en su desarrollo y transformación Adolfo Adrián Álvarez-Rodríguez

\section{ARTÍCULOS}

Organizaciones sociales de pobladores y prácticas de resistencia en contexto de pandemia COVID-19 en Chile

Patricia Castañeda-Meneses

Entre el aislamiento y las brechas digitales: sistematización de experiencia de acompañamiento socioemocional en personas mayores de Temuco, Chile, en tiempos de COVID-19

G. Bernarda Aedo-Neira

Percepción de apoyo social y calidad de vida: la visión de personas mayores chilenas en el contexto de pandemia durante el 2020

Claudia Elena Quiroga-Sanzana

Gabriela Rocío Parra-Monje

Camila Julia Moyano-Sepúlveda

Marco Alejandro Díaz-Bravo

A propósito del qué, cómo y para qué investigar en el campo de la justicia juvenil: La trastienda de un proceso de investigación en Rosario, Argentina Karina De Bella

Proyección social: reflexión sobre una experiencia virtual con adolescentes en época de pandemia en Sucre y Bolivar, Colombia

Claudia Yaneth Martínez-Mina
La intervención social mediante proyectos comunitarios en modalidad virtual en Mérida, México. Una perspectiva desde el Trabajo Social Claudia Isabel Tzec-Puch Amairani Aracelly Ceh-Alvarado Yanet Guadalupe González-Canul

Resignificación de los vínculos Sociales durante la pandemia de COVID-19: narrativas de personas que convergen en Conjuntos residenciales en Bogotá, Colombia

Andrea Marcela Reyes-García

Laura Daniela Molina-Ortiz

La paradoja de la familia doblemente recluida: clínica de una pandemia en Colombia

Norman Darío Moreno-Carmona

Juan José Cleves-Valencia

Ciencias sociales, mundo y pandemia: por un futuro posible

José Miguel Segura-Gutiérrez

Lina Paola Vásquez-Ávila

¿Qué es Trabajo Social? Los primeros vínculos de los estudiantes con la profesión: experiencias de Argentina, Brasil, Paraguay y Uruguay

Silvia Orieta Rivero-Rodríguez

Leonel Del Prado

Nidia Graciela Battilana-Amarilla

Rosilaine Coradini-Guilherme

Intervención profesional de trabajadores sociales de la universidad del Quindio, Colombia. Asuntos epistemológicos, metodológicos y ético-políticos

Ana María Gil-Ríos 
Reflexiones sobre la intervención social en lo rural: experiencias en el Magdalena Medio, Colombia

Claudia Milena Quijano-Mejía

Johana Linares-García

Construcción de rutas de atención integral a la convivencia escolar en Cali, Colombia: sistematización de la experiencia

Leidy Johana Prado-Montaño

Luz Helena López-Rodríguez

Alejandra Gutiérrez-Cárdenas

Experiencia del proceso de intervención familiar de los actores participantes en la Escuela para Familias del ICBF-Jamundí, Colombia Katherin Viviana Silva-Minotta

María José Forero-Izquierdo

Jimena del Pilar Jaramillo-Jaramillo

\section{RESEÑAS DE LIBROS}

Alerta global: políticas, movimientos sociales y futuros en disputa en tiempos de pandemia Jairo Crispín

El neoliberalismo como teología política. Habermas, Foucault, Dardot, Laval y la historia del capitalismo contemporáneo

José Francisco Desentis-Torres

La Reconceptualización del Trabajo Social en Colombia: Análisis histórico-crítico de las décadas de 1960-1970

Juan Pablo Sierra-Tapiro

ARTISTA INVITADO

Juan Camilo González

Prospectiva

\section{PROSPECTIVA}

Revista de Trabajo Social e Intervención Social

No. 33 • ene.-jun. 2022

e-ISSN: 2389-993X • Universidad del Valle 